\title{
BMJ Open Prevalence and incidence of dyslipidaemia among adults in Africa: a systematic review and meta-analysis protocol
}

Jean Jacques N Noubiap, ${ }^{1,2}$ Jobert Richie N Nansseu, ${ }^{3}$ Jean Joel R Bigna, ${ }^{4}$ Ahmadou M Jingi, ${ }^{5}$ André Pascal Kengne ${ }^{6}$

To cite: Noubiap JJN, Nansseu JRN, Bigna JJR, et al. Prevalence and incidence of dyslipidaemia among adults in Africa: a systematic review and meta-analysis protocol. BMJ Open 2015;5:e007404. doi:10.1136/bmjopen-2014007404

- Prepublication history and additional material is available. To view please visit the journal (http://dx.doi.org/ 10.1136/bmjopen-2014007404).

Received 8 December 2014 Revised 16 February 2015 Accepted 25 February 2015

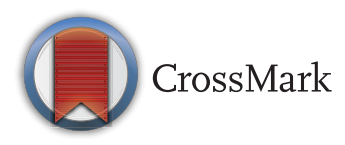

For numbered affiliations see end of article.

Correspondence to Dr Jean Jacques N Noubiap; noubiapjj@yahoo.fr

\section{ABSTRACT}

Introduction: Cardiovascular disease (CVD) is the leading cause of death globally and disproportionately affects low-income and middle-income countries. Dyslipidaemia is an important modifiable risk factor for CVD. There are important knowledge gaps regarding the population levels of lipid variables and frequency of non-optimal levels in populations within Africa. We propose to conduct a systematic review to determine the prevalence and occurrence of dyslipidaemia in adult populations within countries in Africa.

Methods and analysis: We will perform a comprehensive search to identify all possible published and unpublished studies on the prevalence or incidence of dyslipidaemia in Africa reported from 1 January 1980, without language restriction. The scientific databases PubMed MEDLINE, EMBASE and ISI Web of Science will be searched, as well as Grey literature. Following study selection, full-text papers acquisition, and data extraction and synthesis, we will appraise the quality of studies and risk of bias, and assess heterogeneity. Prevalence/incidence data will be summarised by country and geographic regions and a meta-analysis will be conducted for variables defined identically across studies. Variance stabilising transformations will be applied as appropriate to the row data before meta-analysis. This systematic review will be reported according to the MOOSE Guidelines for Meta-Analyses and Systematic Reviews of Observational Studies.

Ethics and dissemination: The current study is based on published data and as such ethics consideration is not a requirement. This review is expected to provide relevant data to help in quantifying the magnitude of dyslipidaemia in African populations, to emphasise the need for appropriate prevention and control strategies, and to identify research gaps and remaining challenges. The final report of the systematic review in the form of a scientific paper will be published in peer-reviewed journals. Findings will further be presented at conferences and submitted to relevant health authorities.

Trial registration number: PROSPERO CRD42014015376.

\section{INTRODUCTION}

Cardiovascular disease (CVD) including stroke, coronary heart disease and peripheral arterial disease is the leading cause of death globally. ${ }^{1}$ An estimated 17.3 million people died from CVD worldwide in 2008, representing $30 \%$ of all deaths in that year. ${ }^{1}$ Of these deaths, about 7.3 million and 6.2 million, respectively, were due to coronary heart disease and stroke. ${ }^{2}$ Furthermore, CVD disproportionally affects low-income and middle-income countries (LMIC), where about $80 \%$ of the global CVD mortality occur. ${ }^{1}$

Dyslipidaemia is defined by the presence of non-optimal levels of blood lipids. In clinical practice guidelines, dyslipidaemia is mostly defined by elevated total cholesterol (TC) and/or low-density lipoprotein cholesterol (LDL-C), but the definition is also often extended to include non-optimal levels of high-density lipoprotein cholesterol (HDL-C), triglyceride (TG), apolipoprotein B and apolipoprotein $\mathrm{A} 1 .^{3}$ Dyslipidaemia is one the most important modifiable risk factors for CVD. ${ }^{4-6}$ According to the WHO, globally, a third of ischaemic heart disease is attributable to high cholesterol. Overall, raised cholesterol is estimated to cause 2.6 million deaths $(4.5 \%$ of total) and 29.7 million disability adjusted life years (DALYs), or $2 \%$ of total DALYs. In 2008, the global prevalence of raised total cholesterol among adults $(\geq 5.0 \mathrm{mmol} / \mathrm{L})$ was $39 \% \quad(37 \%$ for males and $40 \%$ for females) ${ }^{7}$

There is robust evidence that successful treatment of dyslipidaemia substantially reduces morbidity and mortality from CVD. ${ }^{8-11}$ For instance, such treatment can reduce the risk of heart disease by $30 \%$ over 5 years. ${ }^{8}$ Moreover, the benefits of lipid-lowering therapy are not only experienced by individuals with CVD, benefits also extend to individuals without clinically overt CVD. ${ }^{8}$ 
Although the burden of CVD is increasing in developing countries, including those within Africa, critical knowledge gaps on the epidemiology of the disease around the continent remain, including the lack of data regarding the distribution of key risk factors such as dyslipidaemia. We present the protocol for a systematic review to assess the distribution and occurrence of dyslipidaemia among adults within Africa. We are not aware of any previous effort to critically review existing published data on dyslipidaemia in this part of the world.

This protocol is prepared and presented according to the PRISMA-P 2015 guidelines. $^{12}$

\section{OBJECTIVES}

To conduct a systematic review and meta-analysis to determine the prevalence, incidence and characteristics of dyslipidaemia in populations within countries in Africa.

\section{REVIEW QUESTION}

This systematic review will seek to address the following research question:

What are the prevalence and incidence of dyslipidaemia in adult populations within countries in Africa as reported in studies published from 1980 to 2015?

\section{CRITERIA FOR CONSIDERING STUDIES FOR THE REVIEW Inclusion criteria}

1. We will include cross-sectional, case-control or cohort studies of adult participants residing in African countries reporting the prevalence or incidence of dyslipidaemia, or enough data to compute these estimates.

2. Diagnosis of dyslipidaemia will be based on doctor diagnosis, or measured lipid profile.

We will consider all published and unpublished studies reported from 1 January 1980, while accounting for changes in the definition of dyslipidaemia over time. No language restriction will be applied.

\section{Exclusion criteria}

1. Studies conducted among populations of African origin residing outside Africa.

2. Studies not performed in human participants.

3. Studies in subgroups of participants selected on the basis of the presence of dyslipidaemia.

4. Studies in children and adolescents (ie, age $<15$ years).

5. Case series (sample size of less than 50 participants), letters, reviews, commentaries and editorials.

6. Studies lacking primary data and/or explicit method description.

7. Duplicates; for studies published in more than one report, the most comprehensive and up-to-date version will be used.

8. Studies with serious ethical issues.
SEARCH STRATEGY FOR IDENTIFYING RELEVANT STUDIES

The methods for this systematic review have been developed according to the MOOSE Guidelines for Meta-Analyses and Systematic Reviews of Observational Studies. ${ }^{13}$ The search strategy will be implemented in two stages.

\section{Bibliographic database searches}

A. We will perform a comprehensive search of databases to identify all relevant articles published on dyslipidaemia in Africa between January 1980 and February 2015 without language restriction. A systematic search of PubMed MEDLINE, EMBASE and ISI Web of Science (Science Citation Index) will be undertaken using a predefined strategy based on the combination of relevant terms and the names of each of the 54 African countries and African subregions to capture the largest number of studies. We will use text words as well as medical subject heading (MeSH) terms; for example 'dyslipidemia', 'hyperlipidemia', 'lipid disorder', 'hypercholesterolemia' and 'hypertriglyceridemia'. These terms and their variants will be used in varying combinations. The literature search strategy will be adapted to suit each database. The main search strategy is shown in table 1 .

B. We will manually search the reference lists of eligible articles and relevant reviews, and trace their citations using the ISI Web of Knowledge portal. Grey literature, from key conference proceedings and sources including the African regional database 'African Index Medicus', 'OpenSIGLE', the WHO International Clinical Trials Registry, the WHO Global Infobase and the meta-Register of Controlled Trials (mRCT) will also be reviewed for relevant information.

\section{Selection of studies for inclusion in the review}

Two investigators will independently identify articles and sequentially screen their titles and abstracts for eligibility. Full text of articles deemed potentially eligible will be acquired. These investigators will further independently assess the full text of each study for eligibility, and consensually retain studies to be included. Disagreement will be solved by a third assessor. We will use a screening guide to ensure that the selection criteria are reliably applied by all assessors.

\section{APPRAISAL OF THE QUALITY OF INCLUDED STUDIES}

We will evaluate included studies for quality and bias using an adapted version of the Risk of Bias Tool for Prevalence Studies developed by Hoy et $a l^{14}$ (see online supplementary appendix S1), which will be applied to screened full-text articles. Assessment of the risk of selection and attrition bias will use the Cochrane guidelines available in Review Manager V.5.3 (http://tech. cochrane.org/revman). Furthermore, the reporting quality of each study will be assessed using the STROBE 
Table 1 Search history PubMed

\begin{tabular}{|c|c|c|}
\hline Search & Search terms & Hits \\
\hline 1 & $\begin{array}{l}\text { Dyslipidemia [tw] OR lipid disorder [tw] OR lipid [tw] OR hypercholesterolemia [tw] } \\
\text { OR hypertriglyceridemia [tw] OR hyperlipidemia [tw] }\end{array}$ & \\
\hline 2 & Dyslipidemia [MeSH Terms] & \\
\hline 3 & \# 1 OR \# 2 & \\
\hline 4 & 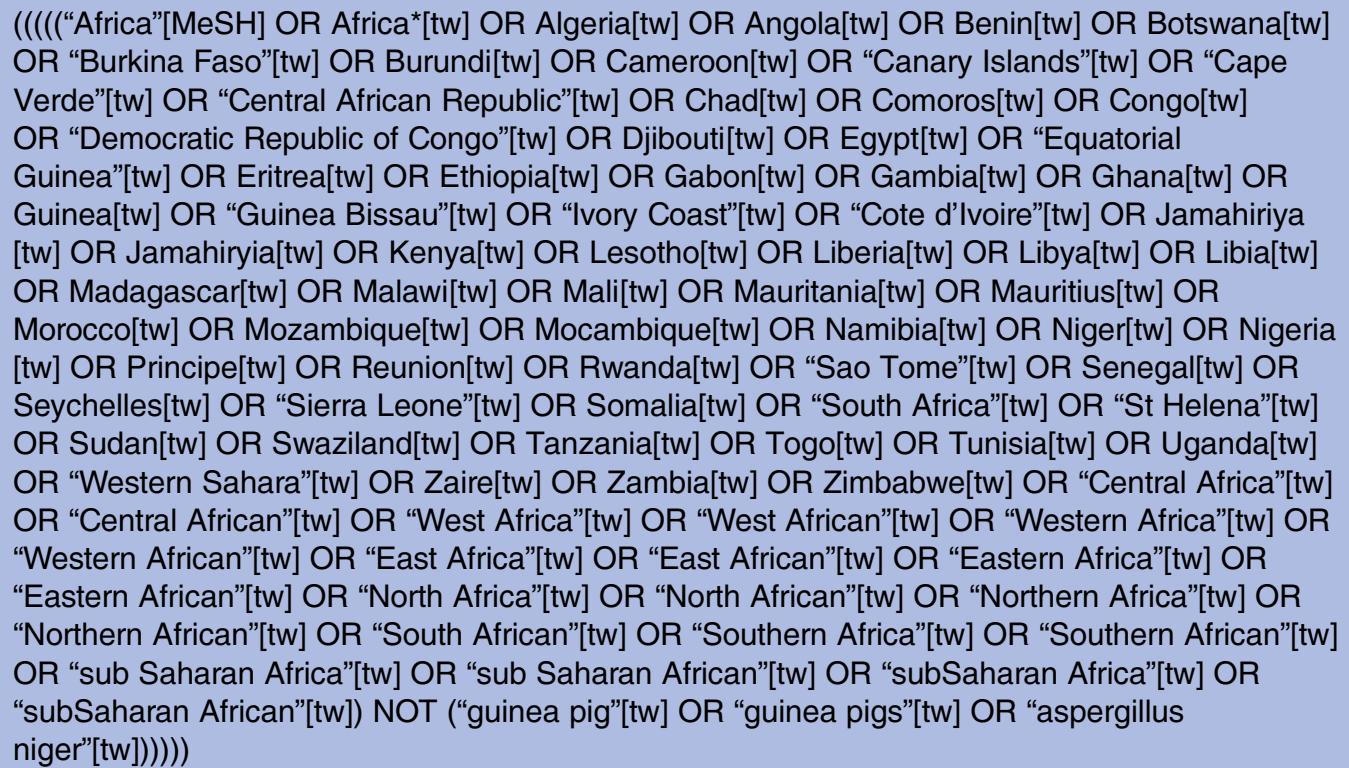 & \\
\hline 5 & \# 3 AND \# 4 & \\
\hline 6 & \#5 Limits: $1980 / 01 / 01$ to $2015 / 02 / 28$ and studies done in Humans & \\
\hline
\end{tabular}

checklist (see online supplementary appendix S2) ${ }^{15}$ Risk of bias and quality scores will be presented in a table.

\section{DATA EXTRACTION AND MANAGEMENT}

Two assessors will independently extract data regarding general information (authors, year, country, type of publication), study characteristics (study design, setting, sample size, response rate, mean or median age and proportions of female participants, diagnosis criteria for dyslipidaemia, possible disease specific to the study population), prevalence and incidence of dyslipidaemia. Where only primary data (sample size and number of outcomes) are provided, these will be used to calculate the prevalence or incidence estimates. Where prevalence/incidence rates or relevance for estimating them are not available, we will contact the corresponding author of the study to request the missing information. Data will be extracted using standardised data collection.

\section{DATA SYNTHESIS INCLUDING ASSESSMENT OF HETEROGENEITY}

Prevalence/incidence data will be summarised by country and geographic region (central, eastern, northern, southern and western Africa). A meta-analysis will be conducted for variables defined identically across studies. SEs for the study-specific estimates will be determined from the point estimate and the appropriate denominators, assuming a binominal (or Poisson for incidence data) distribution. We will pool the study-specific estimates using a random effects meta-analysis model to obtain an overall summary estimate of the prevalence/ incidence across studies, after stabilising the variance of individual studies with the use of the Freeman-Tukey double arc-sine transformation. ${ }^{16}$ Heterogeneity will be assessed using the $\chi^{2}$ test on Cochrane's $Q$ statistic $^{17}$ and quantified by calculating the $\mathrm{I}^{2}{ }^{18}$ Values of $25 \%, 50 \%$ and $75 \%$ for I2 represent, respectively, low heterogeneity, medium heterogeneity and high heterogeneity. We will assess the presence of publication bias using funnel plots and Egger's test. ${ }^{19}$ Where substantial heterogeneity is detected, we will perform subgroup analysis to investigate the possible sources of heterogeneity using the following grouping variables: age group, sex, study setting (rural vs urban; hospital vs community-based), geographical region (central, eastern, northern, southern and western Africa), study quality. We will assess inter-rater agreement for study inclusion using Cohen's $\kappa$ coefficient. ${ }^{20}$ Data will be analysed using the statistical software R (V.3.0.3 (2014-03-04), The R Foundation for statistical computing, Vienna, Austria).

\section{PRESENTING AND REPORTING THE RESULTS}

The study selection process will be summarised using a flow diagram. Reasons for exclusion of studies will be described. This will follow the MOOSE Guidelines for Meta-Analyses and Systematic Reviews of Observational Studies. ${ }^{13}$ Quantitative data will be presented in 
evidence tables of individual studies as well as in summary tables and forest plots where appropriate. We will examine prevalence/incidence by region, country, setting (rural or urban), time period and disease-specific populations depending on the data available. We plan to report on quality scores and risk of bias for each eligible study. This may be tabulated and accompanied by narrative summaries.

\section{CONCLUSIONS}

CVD has reached epidemic proportions in Africa, driven mainly by hypertension, diabetes, obesity and dyslipidaemia. ${ }^{1}$ The challenging first step to address the burden of CVD on the continent is to establish accurate epidemiological data on this condition and its risk factors. We anticipate that this review on dyslipidaemia in Africa will have implications for policy, practice and research. This review is expected to provide relevant data to help in quantifying the magnitude of dyslipidaemia in African populations and emphasise the need for appropriate prevention and control strategies. The review will also determine the characteristics of dyslipidaemia in the African populations, and may help to confirm some unique features of dyslipidaemia previously reported in a population of West Africans and African-Americans. Indeed, low HDL-C with normal triglyceride levels was found to be the most common lipid pattern in West Africans and African-Americans with metabolic syndrome, suggesting that the lipid profile associated with cardiovascular risk in populations of African ethnicity may differ from that in Caucasians. ${ }^{21}$ Furthermore, this review may identify the research gaps and remaining challenges that may form the basis of future studies targeting various aspects of dyslipidaemia to tackle the burden of the disease in African populations.

The main possible limitations of this review could be the scarcity of studies on the subject and the predominance of clinic-based studies and poor quality data, as revealed by previous reviews on chronic non-communicable diseases in Africa, ${ }^{22-24}$ which hampered definitive inferences and drawing relevant conclusions. Data presented would therefore be only general indicators of the epidemiology of dyslipidaemia on the continent.

\section{ETHICS AND DISSEMINATION}

The current study is based on published data and as such ethics consideration is not a requirement. The final report of the systematic review in the form of a scientific paper will be published in peer-reviewed journals. Findings will further be presented at conferences and submitted to relevant health authorities. We also plan to update the review in future to monitor changes and guide health service and policy solutions.

\footnotetext{
Author affiliations

${ }^{1}$ Internal Medicine Unit, Edéa Regional Hospital, Edéa, Cameroon

${ }^{2}$ Medical Diagnostic Center, Yaoundé, Cameroon
}

${ }^{3}$ Sickle Cell Unit, Mother and Child Centre, Chantal Biya Foundation, Yaoundé, Cameroon

${ }^{4}$ Goulfey District Hospital, Goulfey, Cameroon

${ }^{5}$ Faculty of Medicine and Biomedical Sciences, Department of Internal Medicine and Specialties, University of Yaoundé I, Yaoundé, Cameroon ${ }^{6}$ Non-communicable Disease Research Unit, South African Medical Research Council and University of Cape Town, Cape Town, South Africa

Contributors APK and JJNN conceived and designed the protocol, and JJNN drafted the manuscript. JRNN, JJRB and AMJ revised the manuscript for methodological and clinical content. All authors approved the final version.

Funding This research received no specific grant from any funding agency in the public, commercial or not-for-profit sectors.

Competing interests None.

Provenance and peer review Not commissioned; externally peer reviewed.

Open Access This is an Open Access article distributed in accordance with the Creative Commons Attribution Non Commercial (CC BY-NC 4.0) license, which permits others to distribute, remix, adapt, build upon this work noncommercially, and license their derivative works on different terms, provided the original work is properly cited and the use is non-commercial. See: http:// creativecommons.org/licenses/by-nc/4.0/

\section{REFERENCES}

1. World Health Organization. Global status report on non communicable diseases 2010. Geneva, World Health Organization, 2011.

2. World Health Organization. Global atlas on cardiovascular disease prevention and control. Geneva, World Health Organization, 2011.

3. Stone NJ, Robinson JG, Lichtenstein AH, et al. 2013 ACC/AHA guideline on the treatment of blood cholesterol to reduce atherosclerotic cardiovascular risk in adults: a report of the American College of Cardiology/American Heart Association Task Force on Practice Guidelines. Circulation 2014;129(25 Suppl 2): S1-45.

4. Di Angelantonio E, Gao P, Pennells L, et al. Emerging Risk Factors Collaboration. Lipid-related markers and cardiovascular disease prediction. JAMA 2012;307:2499-506.

5. Voight BF, Peloso GM, Orho-Melander M, et al. Plasma HDL cholesterol and risk of myocardial infarction: a Mendelian randomisation study. Lancet 2012;380:572-80.

6. Yusuf S, Hawken S, Ounpuu S, et al. Effect of potentially modifiable risk factors associated with myocardial infarction in 52 countries (the INTERHEART study): case control study. Lancet 2004;364:937-52.

7. World Health Organization. Global Health Observatory data. Cholesterol. http://www.who.int/gho/ncd/risk_factors/cholesterol_ prevalence/en/ (accessed 14 Feb 2015).

8. Grundy SM, Cleeman JI, Merz CN, et al. Implications of recent clinical trials for the National Cholesterol Education Program Adult Treatment Panel III guidelines. J Am Coll Cardiol 2004;44:720-32.

9. Law MR, Wald NJ, Thompson SG. By how much and how quickly does reduction in serum cholesterol concentration lower risk of ischaemic heart disease? BMJ 1994;308:367-72.

10. Baigent C, Blackwell L, Emberson J, et al., Cholesterol Treatment Trialists' (CTT) Collaboration. Efficacy and safety of more intensive lowering of LDL cholesterol: a metaanalysis of data from 170,000 participants in 26 randomised trials. Lancet 2010;376:1670-81.

11. Robinson JG, Smith B, Maheshwari N, et al. Pleiotropic effects of statins: benefit beyond cholesterol reduction? A meta-regression analysis. J Am Coll Cardiol 2005;46:1855-62.

12. Moher D, Shamseer L, Clarke M, et al. Preferred reporting items for systematic review and meta-analysis protocols (PRISMA-P) 2015 statement. Syst Rev 2015;4:1.

13. Stroup DF, Berlin JA, Morton SC, et al. Meta-analysis of observational studies in epidemiology: a proposal for reporting. Meta-analysis Of Observational Studies in Epidemiology (MOOSE) group. JAMA 2000;283:2008-12.

14. Hoy $D$, Brooks $P$, Woolf $A$, et al. Assessing risk of bias in prevalence studies: modification of an existing tool and evidence of interrater agreement. J Clin Epidemiol 2012;65:934-9.

15. von Elm E, Altman DG, Egger M, et al. The Strengthening the Reporting of Observational Studies in Epidemiology (STROBE) statement: guidelines for reporting observational studies. PLoS Med 2007; 4 :e296. 
16. Barendregt JJ, Doi SA, Lee YY, et al. Meta-analysis of prevalence. J Epidemiol Community Health 2013;67:974-8.

17. Cochran WG. The combination of estimates from different experiments. Biometrics 1954;10:101-29.

18. Higgins IP, Thompson SG. Quantifying heterogeneity in a meta-analysis. Stat Med 2002;21:1539-58.

19. Egger M, Davey Smith G, Schneider M, et al. Bias in meta-analysis detected by a simple, graphical test. BMJ 1997;315:629-34.

20. Landis JR, Koch GG. The measurement of observer agreement for categorical data. Biometrics 1977;33:159-74.

21. Sumner AE, Zhou J, DOumatey A, et al. Low HDL-cholesterol with normal triglycerides levels is the most common lipid pattern in West
Africans and Africans Americans with metabolic syndrome: implications for cardiovascular disease prevention. CVD Prev Control 2010;5:75-80.

22. Burgess PI, MacCormick IJ, Harding SP, et al. Epidemiology of diabetes retinopathy and maculopathy in Africa: a systematic review. Diabet Med 2013;30:399-412.

23. Stanifer JW, Jing B, TOlan S, et al. The epidemiology of chronic kidney disease in sub-Saharan Africa: a systematic review and meta-analysis. Lancet Glob Health 2014;2:e174-81.

24. Connor MD, Walker R, Modi G, et al. Burden of stroke in black populations in sub-Saharan Africa. Lancet Neurol 2007;6:269-78. 